\title{
Immunologische und allergologische Aspekte der Gentechnologie
}

\author{
B. M. Stadler \\ Institut für Immunologie, Inselspital, Bern, Schweiz \\ Korrespondenz an: Prof. Dr. Beda M. Stadler, Direktor, Institut für Immunologie, Sahlihaus 2, Inselspital, CH-3010 Bern, Schweiz \\ Eingegangen: 17. September 2007 \\ Online First 21 December 2007
}

Schlüsselwörter: Immunologie, Allergologie, Gentechnologie.

\section{Die Akzeptanz einer neuen Technologie}

Dr. Eduard Jenner hat vor mehr als 200 Jahren eine bahnbrechende neue Technologie, die Impfung, als Manuskript dem englischen König unterbreitet, also sozusagen klammheimlich. Dies war aber der Beginn einer neuen Forschungsdisziplin, der Immunologie. Der Begründer hat damals das Wesentliche dieser Disziplin wohl bloss als Intuition verstanden. Er verwendete das Kuhpockenvirus und begann eine Art Tabubruch, den die Gesellschaft bis heute nicht verkraftet hat.

Es ist verständlich, dass damals die Öffentlichkeit Mühe hatte mit der neuen Technologie, schliesslich wurde tierisches Material auf den Menschen übertragen. Mit dem Ausdruck „Vakzinierung“ hat sich die Kuh als vermeintlicher Tabubruch bis heute in der Terminologie gehalten. Fig. 1 zeigt eine zeitgenössische Abbildung der Protestbewegung und verdeutlicht die Ängste, welche durch diese neue Technologie hervorgerufen wurden. Es existieren heute ähnliche Abbildungen, welche die Ängste der Mitbürger im Zusammenhang mit der Gentechnik darstellen. Ganz speziell hat die Organtransplantation einige Comiczeichner bewegt Mensch-Tier-Hybride darzustellen, um solche Ängste allenfalls „verarbeiten“ zu können. Geblieben ist die Skepsis gegenüber der Impfung. Noch heute gibt es impfkritische Gruppen und gar Organisationen von impfkritischen Ärzten, welche die Impfung mit unwissenschaftlichen und rein emotionalen Argumenten bekämpfen.

Die Transplantationsimmunologie wäre also ein weiteres Gebiet, um dem Titel dieses Vortrags gerecht zu werden. Ich möchte sie hier aber ausklammern. Es ist diejenige immunologische Technologie, die am wenigsten weit fortgeschritten ist und deren Akzeptanz mehr als die anderen immunologischen Fortschritte noch an einem Faden hängt (Yang and Sykes, 2007). In dem Zusammenhang wurde die nackte Maus geradezu zu einem weltweiten und zugleich falschen Paradebeispiel, um Ängste zu schüren. Die "nude mouse", als wichtiges Instrument der immunologischen Forschung, hat kein zelluläres Immunsystem, ist aber auf natürliche Weise entstanden. Diese anti-Gentech-Ikone ist so natürlich wie in der Pflanzenzucht die Mutation vom Pfirsich zur Nektarine. Obwohl unsere Mitbürger auf dem Gebiet der grünen Gentechnologie noch wesentlich empfindlicher reagieren, waren hier für einmal die Abwesenheit von Härchen nicht ausschlaggebend, dass die Nektarine akzeptiert wurde. Die Akzeptanz hängt also manchmal an einem Härchen, wie dies auch am Beispiel eines Ledermantels gezeigt werden kann. Ein Pelzmantel ist im Prinzip ein unrasierter Ledermantel, was hingegen diesem Bekleidungsstück keine Akzeptanz verleiht.

\section{Die aktive Immunisierung}

Impfstoffe gehören zu den ersten gentechnischen Produkten aus dem Gebiet der Immunologie. Rekombinante Impfstoffe wurden zwar von jenen Mitbürgern akzeptiert, die sich vor den Folgen einer möglichen Ansteckung fürchteten, also direkt betroffen waren. Im Wettlauf gegen die Antibiotika haben aber leider die Impfstoffe den Kürzeren gezogen, so dass es heute für bakterielle Infektionen zu wenig Impfstoffe gibt. Bei den antiviralen Impfstoffen gibt es gar Mitbürger, die denken, sie hätten ein Recht auf Ansteckung. Die normale Influenza-Impfung gehört in diese Kategorie. Dank der drohenden Pandemiegefahr werden nun endlich moderne Impfstoffe entwickelt. Es ist zu hoffen, dass dies ebenfalls ein Beitrag ist, um die Impfdisziplin zu erhöhen. Neuere antivirale Impfstoffe auf dem Markt, wie z. B. der anti Hepatitis B Impfstoff, versprechen sogar mehr als einen blossen Schutz vor der Ansteckung. Etwa 0.4\% aller Patienten, die eine Hepatitis B Infektion durchmachen, entwickeln später Leberkrebs. Die Impfung gegen Hepatitis B ist also gleichzeitig eine Impfung gegen Krebs. Neuerdings steht zudem ein Impfstoff gegen Papilloma-Viren zur Verfügung, der Frauen vor Gebärmutterhalskrebs schützt.

Die aktive Impfung als neue Technologie zu bezeichnen wäre falsch. Richtig ist aber, dass diese 200 Jahre alte Technologie bis heute keine allgemeine Akzeptanz gefunden hat. Die Gentechnik hat aber die aktive Impfung massgeblich be- 


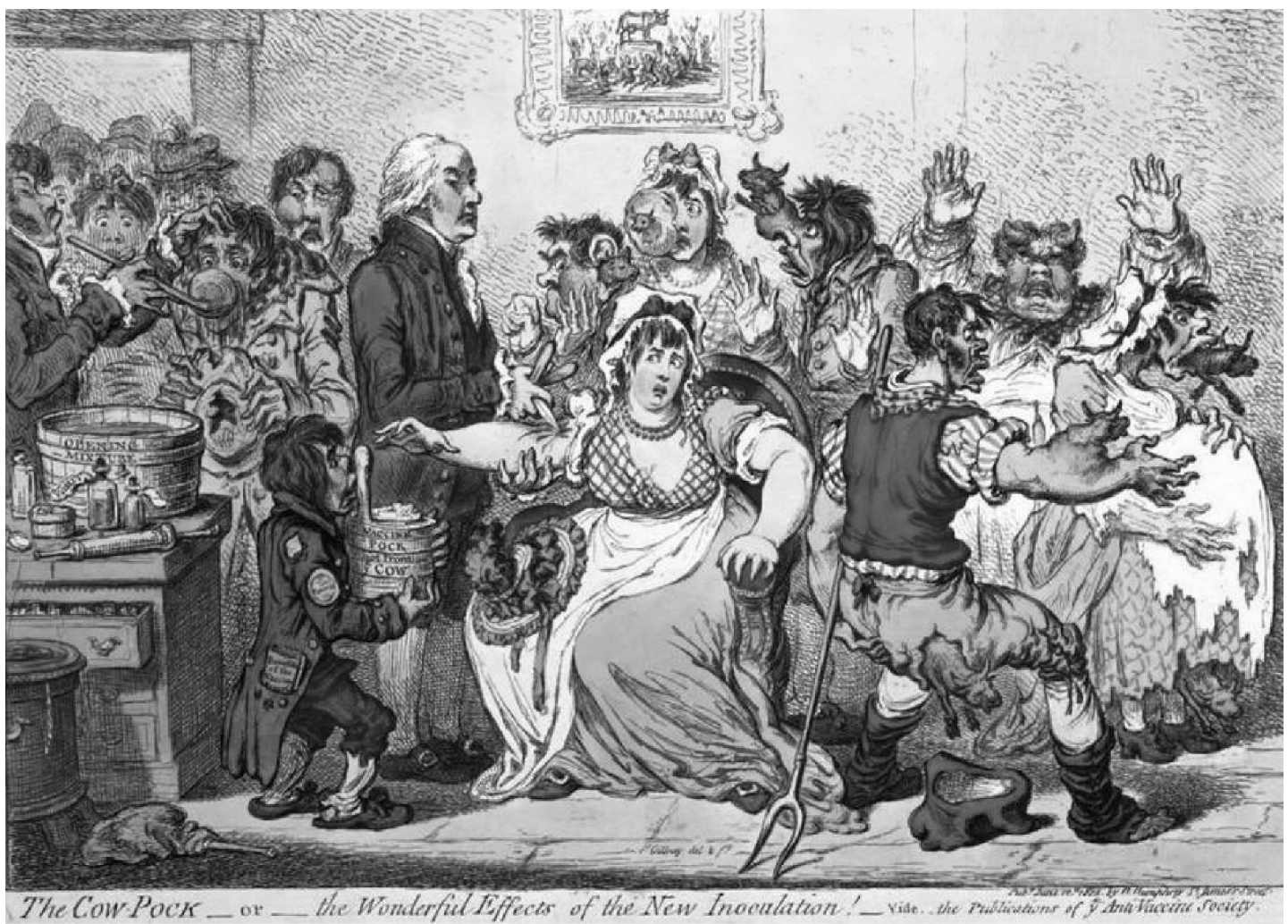

Fig. 1 Hand-kolorierte Zeichnung von James Gillray (1802); publiziert von der britischen Anti-Impf Gesellschaft als Satire über Edward Jenner und "the Wonderful Effects of the New Inoculation“.

fruchtet. Am Horizont stehen für einige Organismen bereits Impfstoffe basierend auf nackter DNA, aber auch rekombinante Bruchstücke von Eiweissen, welche die Immunantwort exakt auf die Schutzfunktion fokussieren. Nicht zuletzt werden die Erkenntnisse aus der Proteomik mit Hilfe von bioinformatischen Verfahren auch weiterhin neue Impfstoffe liefern.

\section{Die passive Immunisierung}

Die aktive Immunisierung führt dazu, dass spezifische Zellen, B-Zellen, generiert werden. Sie produzieren Antikörper, welche vorerst als tierische, später als menschliche Seren zur Therapie eingesetzt wurden. Einige der Antikörper, die heute therapeutisch eingesetzt werden, basieren noch auf den Kinderschuhen einer immunologischen Technologie, nämlich der Hybridomatechnologie. Sie erlaubte grössere Mengen von Antikörpern in vitro herzustellen dank der Fusion von Krebszellen mit Antikörper produzierenden B Zellen. Diese Innovation und die Entdeckung des Genrearrangements waren die Grundlage für den phänomenalen Durchbruch der monoklonalen Antikörper als moderne Therapeutika.

Genmanipulationen gehören zu den Ängsten unserer Bevölkerung. Eine einzelne Genmanipulation in einem Nahrungsmittel führt zu Protestwellen. Erklärt man allerdings einem Laien, das Immunsystem vollführe alleine, um die Vielzahl der verschiedenen Antikörper herzustellen, ca. eine Million Genmanipulationen pro Sekunde, führt dies nicht zu
Akzeptanz, sondern eher zu ungläubigem Staunen. Der Immunologie scheint wiederum die Rolle der Wegbereiterin anheim zu fallen, um darzulegen, wie winzig eigentlich unser Genom ist, und dass selbst unser Proteom fast vernachlässigbar klein ist im Vergleich zur Biodiversität, die vom Immunsystem täglich generiert wird. Ein kleines Säugetier, etwa eine Maus, kann nämlich mehr als $10^{9}$ verschiedenartige Antikörper-Spezifitäten herstellen.

Für die Therapie sind allerdings - je länger je mehr - nur noch rein humane Antikörper von Interesse. Hier zeigt sich ein weiteres Spannungsfeld. Die Öffentlichkeit beginnt erst jetzt mit der Debatte, ob hybride Lebewesen ethisch vertretbar sind; dies ereignet sich, nachdem es schon seit Jahren hybride Zelllinien und gar Mausstämme gibt, die ein humanes Immunsystem tragen. Genau so inkonsequent kommt auch hier wieder der Unterschied zwischen der roten und der grünen Gentechnologie zum Vorschein. Impfstoffe oder rekombinante Antikörper, die in Expressionssystemen hergestellt werden, welche sich im weitesten Sinne an den Menschen anlehnen, sind akzeptiert. Das Gene-Pharming, beispielsweise die Herstellung von Impfstoffen oder Antikörpern in Pflanzen, wartet noch auf Akzeptanz, obwohl die Systeme vorhanden wären und obwohl diese Produktionsweise oft sinnvoller wäre als die gängigen Produktionsmethoden (Floss et al., 2007). 


\section{Produkte und das Gesundheitssystem}

Mehr als 150 Antikörper warten in der Pipeline der Industrie und man beginnt zu realisieren, wie teuer dieser rekombinanten Eiweisse sind. Sie stellen bereits heute ein finanzielles Problem für unser Gesundheitssystem dar. Auch hier zeichnet sich auf dem Gebiet der Immunologie eine erste Trendwende ab. Antikörper bestehen nämlich aus zwei Eiweissketten, die über Disulfid-Brücken verbunden werden. Beide Eiweissketten sind relativ gross und sind somit ein Problem für die Grossproduktion. Eine Verbilligung des Produktionsprozesses könnte für ein günstigeres Produkt sorgen und falls man es zugleich schaffen würde, die Logistik rund um biotechnisch hergestellte Eiweisse zu vereinfachen, z. B. kein Bedarf für eine Kühlkette, könnten diese neuartigen und z.T. sehr effizienten Medikamente auch der Dritten Welt zur Verfügung gestellt werden.

Als ein Beispiel in dieser Richtung dienen Designed Ankyrin Repeat Proteins, kurz DARPins (Stumpp und Amstutz, 2007). Ankyrine sind Repeat-Proteine, die wie Antikörper zu den Bindungsproteinen gehören. Man hat diese grosse und weit verbreitete Proteinfamilie dazu verwendet, künstliche Antikörper herzustellen. Die neuartigen Kunstmoleküle sind nicht nur stabiler, sondern in einfachen Expressionssystemen wie Bakterien günstig zu produzieren. Dazu kommt der Vorteil, dass dieses Verfahren eine grössere Diversität repräsentiert. Die bisher angewendeten gentechnischen Verfahren zur Antikörperherstellung kamen maximal auf eine Vielfalt von $10^{8}$ Spezifitäten. Wie wir oben gesehen haben, entspricht dies in etwa $10 \%$ der der potentiellen Vielfalt von möglichen Antikörpern in einer Maus. Mit DARPins kommt man allerdings spielend auf eine Diversität von $10^{12}$, d.h. diese Moleküle sind nicht nur als neue „klebrige“ Eiweisse zu verwenden, wie die Antikörper, sondern sie könnten genau so gut auch als Antigene dienen.

Diese Möglichkeit ist bei Immunologen längst bekannt und wird als anti-idiotypisches Netzwerk bezeichnet. Es besagt, dass es gegen jeden denkbaren Antikörper auch einen Antikörper gibt, der diesen zu erkennen vermag. Antiidiotypische Antikörper, die Antikörper an der Bindungsstelle erkennen, sehen somit theoretisch gleich aus wie das Antigen. Dieses Prinzip birgt nun die Möglichkeit in sich, dass mittels DARPins ebenfalls ein Teil der Antigene kopiert werden kann (Vogel et al., 2007). Wir wären somit wieder am Ursprung, bei der aktiven Immunisierung, angelangt.

\section{Allergien und gentechnisch veränderte Organismen}

Die Anti-Gentechnik-Protestindustrie arbeitet gerne mit der Angst vor Allergien. Eigentlich ist dies ein absurdes Argument, weil gerade auf dem Gebiet der Allergologie signifikante Erkenntnisfortschritte erzielt wurden. Auch die Geschichte lehrt das Gegenteil. Das viel zitierte Beispiel, als ein Methionin-reiches 2S Albumin aus der Paranuss in Soja kloniert wurde und sich herausstellte, dass das Albumin aus der Paranuss ein Allergen ist, diente dazu, die Ängste weiter zu schüren. Genau dieses Paradebeispiel zeigt aber, dass die Möglichkeit eine allfällige Allergenizität vorherzusagen, bereits in der Vergangenheit funktioniert hat, obwohl damals die Vorhersagemöglichkeiten noch nicht so präzise waren wie heute. Die bestehenden Regulative verhinderten, dass überhaupt ein Feldversuch unternommen wurde.

Die molekularen Strukturen der allermeisten klinisch relevanten Allergene sind heute bekannt und ihre Gensequenzen sind öffentlich zugänglich. Dies ermöglichte uns, einen bioinformatischen Algorithmus zu verwenden, um mit grosser Genauigkeit vorherzusagen, ob ein transgenes Eiweiss ein Allergen sein könnte (Stadler und Stadler, 2005). Der Algorithmus kann praktisch alle Allergene vorhersagen (Marti et al., 2007).

Dieser bioinformatische und eigentlich theoretische Ansatz hat aber noch weitere Informationen geliefert. Die klinische Beobachtung, dass nur eine geringe Anzahl von Eiweissen effektiv allergen ist, hat sich bewahrheitet. Wir finden zurzeit wesentlich weniger als 100 molekulare Strukturen, die vom Körper als Allergen erkannt werden können. Gemessen an der Vielzahl der Eiweisse (in den Proteomen unserer Nahrungsmittel und in allen Organismen, die uns umgeben) ist dies eine verschwindend geringe Anzahl von Eiweissen, die überhaupt ein allergenes Potential aufweisen. Man darf somit behaupten, dass eine Vorhersage der Allergenität möglich ist. Das Argument, gentechnisch veränderte Produkte könnten vermehrt zu Allergien führen, hat somit keine wissenschaftliche Basis.

Selbst potentiell allergene Strukturen sind nicht immer klinisch relevant. Tropomyosin ist ein derartiges Allergen, dessen Grundstruktur von Bakterien über die meisten Organismen bis zu uns konserviert wurde und allergen wirken kann. Kleine Sequenzunterschiede zwischen den verschiedenen Tropomyosinen aus verschiedenen Spezies bedingen, ob das Eiweiss mehr oder weniger allergen ist. Rein theoretische wäre es denkbar, aus Langusten (Shrimp) ein hypoallergenes Nahrungsmittel zu machen, indem bovines Tropomyosin in diese hinein kloniert würde anstelle des eigenen Tropomyosins. Aus einem ehemaligen allergenen und somit gefährlichen Nahrungsmittel entstünde ein hypoallergenes und sicheres Nahrungsmittel (Reese et al., 2005). Noch naheliegender wäre natürlich, das menschliche Tropomyosin in die Shrimps zu klonieren gefolgt von einem gesellschaftlichen Aufschrei, genau wie damals, als Dr. Jenner mit Kuhmaterial den Menschen geimpft hat, um die Pocken zu verhindern.

\section{Literatur}

Floss, D. M., Falkenburg, D. und Conrad, U. (2007) Production of vaccines and therapeutic antibodies for veterinary applications in transgenic plants: Transgenic Res 16: 315-332.

Marti, P., Truffer, R., Stadler, M. B., Keller-Gautschi, E., Crameri, R., Mari, A., Schmid-Grendelmeier, P., Miescher, S. M., Stadler, B. M. und Vogel, M. (2007) Allergen motifs and the prediction of allergenicity. Immunol Lett 109: 15 47-55.

Reese, G., Viebranz, J., Leong-Kee, S. M., Plante, M., Lauer, I. und Randow, S. (2005) Reduced allergenic potency of VR9-1, a mutant of the major shrimp allergen Pen a 1 (tropomyosin). J Immunol 175: 8354-8364. 
Stadler, M. B. und Stadler, B. M. (2003) Allergenicity prediction by protein sequence. FASEB J. Journal express article 10.1096/fj.021052 fje. Published online April 22, 2003, and 17: 1141-1143.

Stumpp, M. T. und Amstutz, P. (2007) DARPins: a true alternative to antibodies. Curr Opin Drug Discov Devel 10: 153-159.

Vogel, M., Keller-Gautschi, E., Baumann, M. J., Amstutz, P., Ruf, C.,
Kricek, F. und Stadler, B. M. (2007) Designed ankyrin repeat proteins as anti-idiotypic-binding molecules. Ann N Y Acad Sci 1109: 9-18.

Yang, Y. G. und Sykes, M. (2007) Xenotransplantation: current status and a perspective on the future. Nat Rev Immunol 7: 519-531.

To access this journal online:

http://www.birkhauser.ch/JVL 